\title{
Comunidades fitoplanctónicas indicadoras del estado trófico en las Playas del Este, La Habana, Cuba
}

\section{Phytoplankton communities as indicators of the trophic status of Playas del Este, La Habana, Cuba}

\author{
Claudia Bustamante López ${ }^{1 *}$, Mayelín Carmenate Fernández ${ }^{2}$, Gladys Margarita Lugioyo ${ }^{2}$ y \\ Sandra Loza Álvarez ${ }^{2}$
}

\begin{abstract}
RESUMEN
En los ecosistemas costeros los análisis del fitoplancton permiten pronosticar cambios del estado trófico. Este trabajo se propuso caracterizar la estructura y composición de comunidades fitoplanctónicas como indicadoras del estado trófico y describir variaciones interanuales y espaciales del fitoplancton relacionadas con factores abióticos en las Playas del Este. Se ubicaron 11 estaciones y se determinó la estructura fitoplanctónica a partir de muestras de fitoplancton de red y de botella. Los flagelados y el género Navicula sp. mantuvieron una presencia espacio-temporal constante en la etapa muestreada. Otros géneros y especies que también mostraron una alta frecuencia de aparición en febrero y julio del año 2011 y febrero y agosto del año 2012 fueron: Scrippsiella trochoidea y Gymnodinium sp. Se detectaron organismos potencialmente nocivos como: Oscillatoria sp., S. trochoidea, Akashiwo sanguinea, Alexandrium sp. y Gambierdiscus sp., sobre todo en los meses de febrero de ambos años. La contribución por grupos a la comunidad fitoplanctónica en ambos años mostró un predominio de los flagelados (más del 50\%), con un escaso aporte de diatomeas y dinoflagelados al total de la comunidad (menos del 25\%). La principal afectación al desarrollo fitoplanctónico se debe a la disponibilidad de nitrógeno y fósforo. Las estaciones Tropicoco, Tarará y Bacuranao son las de mayor densidad fitoplanctónica para ambos años. Existió poca diferenciación entre épocas del $2011(R=0.465)$ y $2012(R=0.212)$ y estaciones por cada período climático $(R=0.324)$. El estado trófico de las aguas se clasificó en los años 2011 y 2012 como oligomesotróficas.
\end{abstract}

Palabras claves: Comunidad fitoplanctónica, estado trófico, diatomeas, dinoflagelados, Playas del Este.

\begin{abstract}
The analysis of phytoplankton in coastal ecosystems helps predict changes in trophic status. This paper is aimed at characterizing the structure and composition of phytoplankton communities as indicators of trophic state and describing inter-annual and spatial variations of phytoplankton related to abiotic factors in Playas del Este. Eleven stations were located and the phytoplankton structure was determined from samples of network and bottle phytoplankton. Flagellates and Navicula genus held a constant spatiotemporal presence in the sampled period. Other genus and species that also showed high frequency in February and July 2011 and February and August 2012 were: Scrippsiella trochoidea and Gymnodinium sp. Potentially harmful organisms were also detected such as Oscillatoria sp., S. trochoidea, Akashiwo sanguinea, Alexandrium sp. and Gambierdiscus sp., mainly in the month of February for both years. The contribution by groups to the phytoplankton community in both years showed predominance of flagellates (over 50\%) and a small contribution of diatoms and dinoflagellates in the total community (less than $25 \%$ ). The availability of nitrogen and phosphor is the main

\footnotetext{
1 Centro de Investigaciones de Ecosistemas Costeros (CIEC), Ave. de los Almácigos, Cayo Coco, Ciego de Ávila, Cuba. algas@.ciec.cu*

2 Instituto de Oceanología (IDO), Ave 1ra No. 18406 e/ 184 y 186, Playa, La Habana, Cuba. mayelin@oceano.inf.cu, margarita@oceano.inf.cu, sandra@oceano.inf.cu
}

Recibido: 11 de enero de 2016

Corregido: 13 de junio de 2016

Aceptado: 13 de junio de 2016

DOI: http://dx.doi.org/10.15359/revmar.8-2.5 
element affecting the development of phytoplankton. The Tropicoco, Tarará, and Bacuranao stations have the highest phytoplankton density during both years. Insignificant differences were detected between the 2011 ( $R$ $=0.465)$ and $2012(\mathrm{R}=0.212)$ seasons and climate stations for each period $(\mathrm{R}=0.324)$. The trophic status of waters was classified as oligo-mesotrophic for 2011 and 2012.

Keywords: Phytoplankton community, trophic status, diatoms, dinoflagellates, Playas del Este.

\section{INTRODUCCIÓN}

Los impactos producidos por la actividad humana pueden provocar alteraciones de las condiciones ambientales de la zona costera. Por otro lado, entre los múltiples indicadores biológicos que permiten pronosticar los posibles cambios del estado trófico de los ecosistemas marinos y costeros, resultan particularmente útiles los análisis de la comunidad fitoplanctónica, debido a la rapidez y magnitud de su capacidad de respuesta ante las perturbaciones ambientales (De la Lanza-Espino et al. 2000; Moreira et al. 2007).

De acuerdo con la naturaleza de los contaminantes, se favorecen cambios en la diversidad y la abundancia de las especies de fitoplancton. En el ámbito comunitario, se pueden manifestar alteraciones en la estructura, y en el ámbito específico, la presencia o desaparición de especies sensibles y/o el desarrollo de especies nocivas y/o tóxicas (Bonilla \& Conde, 1998; Loza et al. 2003) que pueden provocar cambios de coloración de las aguas y afectaciones a la salud de los usuarios.

Cabrera \& Suárez (2003) plantean que las microalgas constituyen un grupo focal de gran importancia para el funcionamiento de los ecosistemas marinos y costeros, al ser estas indicadoras de contaminación orgánica por responder a la calidad de las aguas en que habitan, o simplemente por constituir un componente esencial en las tramas tróficas. Su estudio desde el punto de vista taxonómico es el punto de partida obligado para la interpretación de las condiciones ecológicas imperantes en la zona costera.

No todas las especies de fitoplancton existentes en los ecosistemas marinos expuestos a procesos eutróficos presentan las mismas características de tolerancia al cambio de la concentración de nutrientes, este proceso usualmente va acompañado de una reducción de la diversidad y se reduce la equitatividad de la comunidad en un aparente control de tipo bottom-up (Fretwell \& Barach, 1977; Achá \& Fontúrbel, 2003), donde tienden a predominar ciertos grupos eurioicos con la reducción y/o desaparición de otros, afectando así al conjunto de la comunidad.

En este sentido, dependiendo de la naturaleza de los contaminantes, se presentan ciertos grupos característicos en los ecosistemas acuáticos como las diatomeas, los dinoflagelados y las cianobacterias, que se han manifestado como buenos bioindicadores para evaluar la calidad del agua y los cambios ambientales (Vaulot et al. 2008; Ebenezer et al. 2012).

Un ejemplo de esta situación es la existencia de una fuerte relación entre 
las diatomeas y las aguas que poseen una elevada disponibilidad de compuestos inorgánicos como los silicatos, nitratos y fosfatos (Barron \& Baldauf, 1989) que estimulan su desarrollo, ya que constituyen un grupo estrictamente autótrofo (Hasle \& Syvertsen, 1996). Los dinoflagelados, por su parte, presentan una gran sensibilidad a las variaciones del ambiente, por lo que se les usa como indicadores biológicos de algunos parámetros ambientales (salinidad, temperatura y movimientos de las masas de agua) (GamboaMárquez et al. 1994). Por otra parte, la proliferación de cianobacterias es una de las alteraciones comúnmente asociadas a la eutrofización. Esta puede tener un profundo efecto sobre la estructura comunitaria del plancton.

Debido a que es el principal pigmento fotosintético presente en las algas, la concentración de clorofila $a$ es otro de los indicadores que se utiliza para estimar en forma indirecta la biomasa de las comunidades fitoplanctónicas (Gregor \& Marsálek, 2004). Este pigmento también constituye un indicador del grado de contaminación de los ecosistemas acuáticos y un importante índice del estado fisiológico del fitoplancton (Pinto et al. 2001).

Las Playas del Este constituyen uno de los principales polos turísticos de Cuba y el balneario de sol y playa más importante de La Habana (Ruiz \& Delgado, 2012). El desarrollo acelerado de esta región turística en los últimos años, el crecimiento, la expansión poblacional y económica y el aumento de la industrialización ejercen una gran presión sobre esta zona costera de forma proporcional.

Garantizar el aprovechamiento sostenible de las playas de la región turística de las Playas del Este, es primordial para ofertar este producto con mayor calidad y así poder elevar su valor añadido. Teniendo en cuenta que esta zona costera se encuentra directamente afectada por innumerables actividades de origen antrópico, este trabajo se propone como objetivos caracterizar la estructura y composición de las comunidades fitoplanctónicas como indicadoras de estado trófico, así como describir las variaciones interanuales y espaciales del fitoplancton relacionadas con los factores abióticos.

\section{MATERIALES Y MÉTODOS}

El área de estudio comprende el sector costero entre playa Bacuranao y el Rincón de Guanabo que se encuentra ubicado en la Ensenada de Sibarimar, al este de la capital habanera. Presenta $15.6 \mathrm{~km}$ de litoral, con una zona costera que tiene una extensión de playa de 12 $\mathrm{km}$. Para este estudio se muestrearon un total de 11 estaciones, tomando en cuenta las unidades geomorfológicas establecidas por Sosa et al. (2005): zonas de afluencia de bañistas y focos de contaminación (Fig. 1).

Los muestreos se realizaron en un período de dos años (2011-2012), en época poco lluviosa que comprendió el mes de febrero para ambos años y en época lluviosa que comprendió el mes de julio para el 2011 y el mes de agosto 


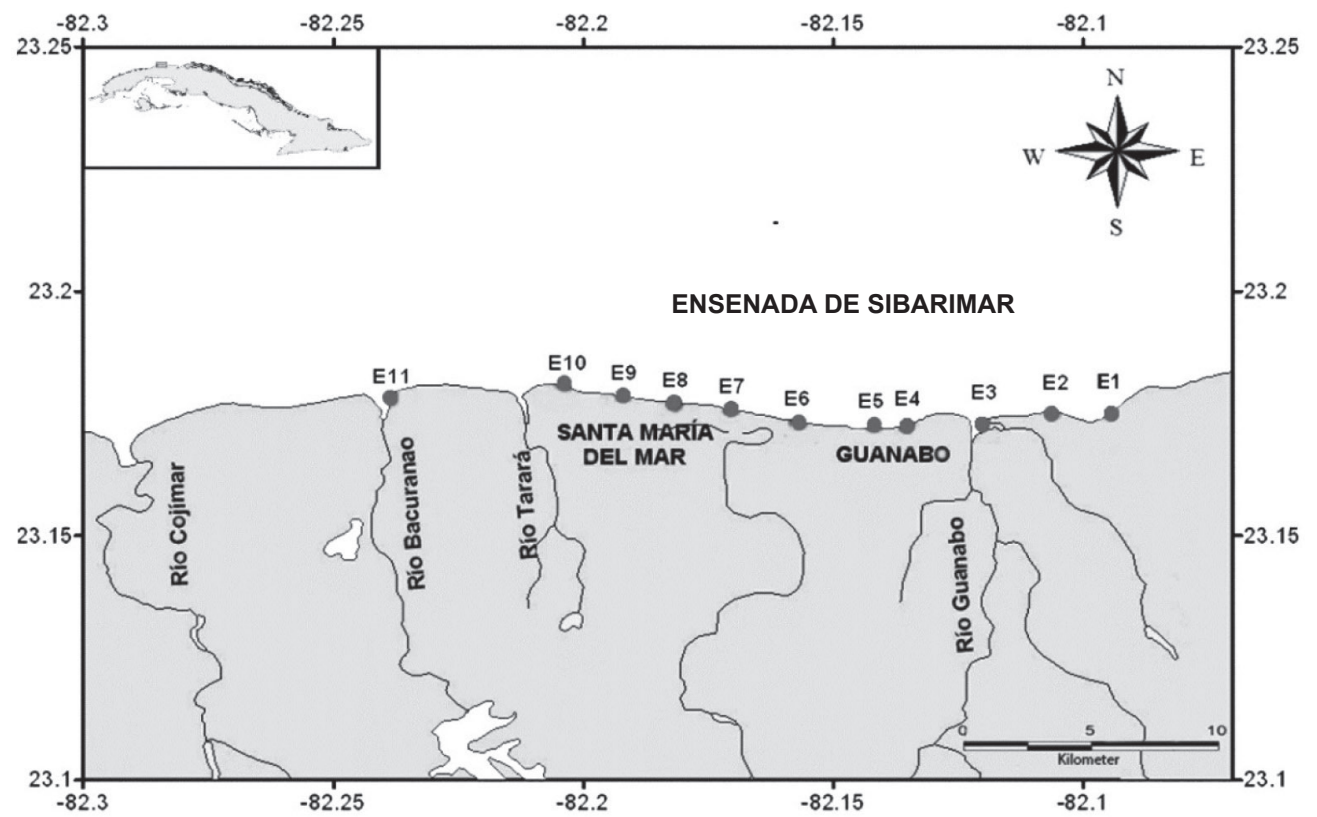

Fig. 1. Estaciones de muestreo (E.1: Rincón de Guanabo, E.2: Ranchón de Brisas del Mar, E.3: Guardafronteras, E.4: Agro Guanabo, E.5: Calle 476-478, Guanabo, E.6: Boca Ciega, E.7: Mi Cayito, E.8: Hotel Tropicoco, E.9: El Mégano, E.10: Playa Tarará, E.11: Bacuranao)

Fig. 1. Sampling stations (E.1: Rincón de Guanabo, E.2: Ranchón de Brisas del Mar, E.3: Guardafronteras, E.4: Agro Guanabo, E.5: Street 476-478, Guanabo, E.6: Boca Ciega, E.7: Mi Cayito, E.8: Tropicoco Hotel, E.9: El Mégano, E.10: Tarará Beach, E.11: Bacuranao)

para el 2012. Las muestras se tomaron a los $3 \mathrm{~m}$ de la zona intermareal, durante el período de marea baja, siempre en horarios de la mañana.

La estructura fitoplanctónica se determinó mediante los análisis cualitativo y cuantitativo de la comunidad; para esta evaluación se emplearon muestras de fitoplancton de red y de botella, respectivamente (Miravet et al. 2009). El análisis cualitativo se realizó a partir de la recolecta de agua superficial $(30 \mathrm{~cm})$, mediante arrastres horizontales con una red de $30 \mathrm{~cm}$ de diámetro y 125 $\mu \mathrm{m}$ de abertura de malla. Luego las muestras se preservaron con formol al 4\%. Para el análisis cuantitativo, las muestras se recolectaron en botellas de $1 \mathrm{~L}$ de capacidad y se fijaron con lugol ácido, según Popowski \& Borrero (1989). Las muestras de red y de botella fueron procesadas en el Laboratorio de Microbiología y Necton del Instituto de Oceanología de acuerdo con la metodología propuesta por Miravet et al. (2009); el análisis de ambas muestras de agua consta de varios pasos: preservación, almacenamiento, sifoneo y centrifugación. Las muestras fueron almacenadas al menos durante una semana para la sedimentación de 
las células muertas. Posteriormente, la identificación y el conteo se realizaron con ayuda de un microscopio biológico Karl Zeiss, con un aumento de $400 \mathrm{x}$.

Con el fin de establecer las diferentes categorías taxonómicas se adoptó el sistema de clasificación según el sitio web: www.algaebase.org (Guiry \& Guiry, 2012). La identificación de los taxones se realizó según Licea et al. (1995); Moreno et al. (1996); Tomas (1997) y Sant'Anna et al. (2005).

$\mathrm{Se}$ estimaron los índices ecológicos de Diversidad (Shannon, H), Dominancia (D) y Equitatividad (J) para determinar cambios espaciales y temporales en la comunidad fitoplanctónica; para esto se utilizó el programa Past 3.1 (Hammer et al. 2001) (Cuadro 1).

Se utilizaron los datos de las variables hidroquímicas medidas en los sitios muestreados (Cuadro 2), como demanda química de oxígeno (DQO), oxígeno disuelto (OD), nitrógeno total (NT), fósforo total (PT), fosfato $\left(\mathrm{PO}_{4}\right)$, amonio $\left(\mathrm{NH}_{4}\right)$, nitrógeno inorgánico $\left(\mathrm{NO}_{2}+\mathrm{NO}_{3}\right)$, proporcionados por el Laboratorio de Oceanografía Química del Instituto de Oceanología y obtenidos mediante los protocolos descritos en dicho laboratorio (Montalvo et al. 2013).

El estado trófico se evaluó empleando como indicador la concentración fitoplanctónica. Para ello se utilizó el índice propuesto por Loza (2004) para las aguas de la plataforma cubana, el cual se elaboró a partir de los datos históricos, mediante el empleo de la escala de Frontier (1981) y teniendo en cuenta el índice trófico de Koblentz-Mishke \& Vedernikov (1979).

Se realizaron pruebas de Homogeneidad de Varianzas y de Normalidad y se procedió a efectuar las consiguientes transformaciones de los datos, para la aplicación de pruebas paramétricas. Debido a que, en general, la naturaleza de los parámetros microbiológicos no cumple las premisas, se prosiguió al empleo de pruebas no paramétricas. Se realizó una correlación por Rangos de Spearman para un nivel de significación de 95\%. Se efectuó un análisis de componentes principales (PCA), con los datos de las diferentes variables fisicoquímicas, la clorofila

Cuadro 1. Valores de los índices ecológicos para el 2011 y el 2012 entre los diferentes grupos de la comunidad fitoplanctónica

Table 1. Values of the 2011 and 2012 ecological indexes between the different groups in the phytoplankton community

\begin{tabular}{cccccc}
\hline Índice & Diatomeas & Dinoflagelados & Cianobacterias & Flagelados & $\begin{array}{c}\text { FITO } \\
\text { TOTAL }\end{array}$ \\
\hline Dominancia (D) & 0.5068 & 0.5191 & 0.5153 & 0.5018 & 0.5023 \\
Shannon (H) & 0.6879 & 0.6857 & 0.6859 & 0.6923 & 0.6912 \\
Equitatividad (J) & 0.9924 & 0.9893 & 0.9895 & 0.9988 & 0.9972 \\
\hline
\end{tabular}


Cuadro 2. Valores promedios de las variables fisicoquímicas medidas durante los muestreos del 2011 y 2012

Table 2. Average values of the physicochemical variables measured during the 2011 and 2012 surveys

\begin{tabular}{ccccc}
\hline Variables & febrero del 2011 & julio del 2011 & febrero del 2012 & agosto del 2012 \\
\hline DQO $(\mathbf{m g} / \mathbf{L})$ & $0.82 \pm 0.31$ & $1.64 \pm 0.53$ & $7.55 \pm 1.56$ & $0.97 \pm 0.13$ \\
$\mathbf{N H}_{4}(\boldsymbol{\mu m o l} / \mathbf{L})$ & $0.21 \pm 0.61$ & $0.23 \pm 0.17$ & $0.90 \pm 0.45$ & $0.98 \pm 0.39$ \\
$\mathbf{N T i}(\boldsymbol{\mu m o l} / \mathbf{L})$ & $1.54 \pm 2.42$ & $1.93 \pm 0.60$ & $1.56 \pm 1.54$ & $1.95 \pm 0.48$ \\
$\mathbf{N T}(\boldsymbol{\mu m o l} / \mathbf{L})$ & $27.05 \pm 15.27$ & $22.26 \pm 5.94$ & $70.02 \pm 16.32$ & $23.18 \pm 7.20$ \\
$\mathbf{N T i} / \mathbf{P O}_{4}(\boldsymbol{\mu m o l} / \mathbf{L})$ & $14.8 \pm 12.3$ & $16.7 \pm 22.1$ & $17.9 \pm 18$ & $23.4 \pm 10.3$ \\
$\mathbf{N T} / \mathbf{P T}(\boldsymbol{\mu m o l} / \mathbf{L})$ & $99.1 \pm 95.7$ & $43.4 \pm 14.2$ & $288 \pm 185.1$ & $114 \pm 83.8$ \\
Clorofila $\boldsymbol{a}\left(\mathbf{m g} / \mathbf{L}^{3}\right)$ & $0.26 \pm 0.29$ & $0.68 \pm 0.46$ & $0.02 \pm 0.02$ & $0.01 \pm 0.01$ \\
\hline
\end{tabular}

a y como variable suplementaria la abundancia de fitoplancton total. Posteriormente, se aplicaron un Escalado Multidimensional no Métrico (nmMDS) y un Análisis de Similitud (ANOSIM), cruzado de dos vías, sin réplicas, para determinar diferencias entre las estaciones de muestreo.

Para la confección del mapa se utilizó el programa MAPINFO 6.5 (MapInfo Corp., 2001) con una escala de 1:25 000. Para la ejecución de los gráficos y cálculos de análisis de varianza se utilizó STATISTICA 6 (STATSOFT, 2001).

\section{RESULTADOS}

\section{Composición cualitativa y cuantitativa de la comunidad fitoplanctónica}

La comunidad fitoplanctónica en los meses de febrero y julio del año 2011 estuvo representada por cuatro grupos taxonómicos con un total de 69 taxones, distribuidos en 37 formas de diatomeas, 15 dinoflagelados, 16 cianobacterias y 1 flagelado, mientras que en los meses de febrero y agosto del 2012 se observó un incremento de 125 taxones ( 71 pertenecen al grupo de las diatomeas, 24 a los dinoflagelados, 29 a las cianobacterias y 1 flagelado). En general, el grupo de las diatomeas fue el que más cantidad de especies aportó al total de la comunidad, en los muestreos de los años 2011 y 2012.

El grupo de los flagelados y el género Navicula sp. mantuvieron una presencia espacio-temporal constante en ambas épocas climáticas de los dos años de muestreo. Algunos géneros $y$ especies estuvieron presentes en la mayoría de las estaciones muestreadas con respecto al total de especies encontradas en todos los muestreos, como son: el dinoflagelado Scrippsiella trochoidea con una frecuencia relativa de $41 \%$ y $64 \%$ y el género Gymnodinium (64\% y $91 \%$ ), en el año 2011 y en el año 2012, respectivamente. 

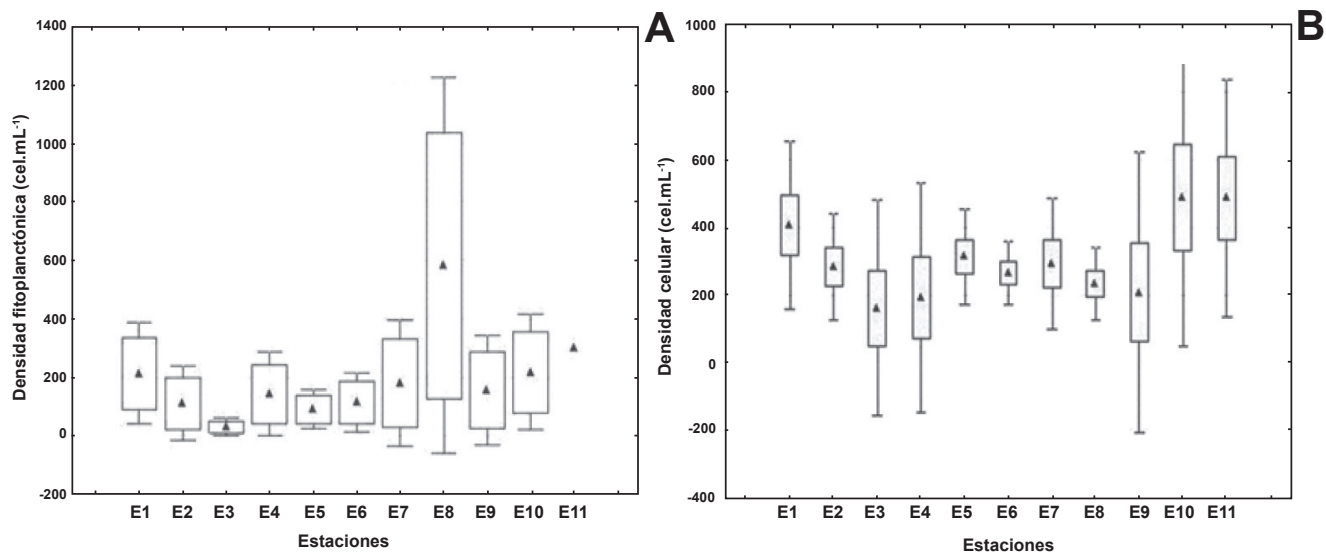

Fig. 2. Comportamiento de la densidad fitoplanctónica durante los años 2011 (B) y 2012 (A) en ambas temporadas climáticas

Fig. 2. Behavior of the phytoplankton density during 2011 (B) and 2012 (A) in both climatic seasons

Se detectaron organismos potencialmente nocivos, entre los que se encuentran Oscillatoria sp., S. trochoidea, Akashiwo sanguinea, Alexandrium sp. y Gambierdiscus sp., representados fundamentalmente en la época poco lluviosa de ambos años (2011 y 2012), que no mostraron mayor abundancia relativa $(3 \%$ del total de la comunidad fitoplanctónica) en relación con las restantes especies de la comunidad fitoplanctónica. En las estaciones Boca Ciega (E.6), Playa Tarará (E.10) y Bacuranao (E.11) se encontró el mayor número de especies nocivas por estación.

Las concentraciones del fitoplancton total en los dos meses muestreados de ambos años fluctuaron entre 9.00 y 3717 cél.mL $\mathrm{mL}^{-1}$. Los mayores valores se hallaron en el 2012 $\left(301.56 \pm 150.01\right.$ cél. $\left.\mathrm{mL}^{-1}\right)$, siendo Tarará (E.10) la estación de mayor concentración $(488.40 \pm 220.90$ cél. $\mathrm{mL}^{-1}$ ) (Fig. 2B).
La evaluación por época mostró que los mayores valores de concentración de fitoplancton total se obtuvieron en el período lluvioso del año 2012 (313.20 \pm 76.31 cél. $\mathrm{mL}^{-1}$ ), siendo la estación más representativa la del Rincón de Guanabo (E.1) (70\%). Los menores se obtuvieron en la época poco lluviosa del 2011 (54.91 \pm 33.76 cél. $\left.\mathrm{mL}^{-1}\right)$, donde el mayor aporte se encontró en la estación Tropicoco (E.8) (64\%). Se observó que las concentraciones en la época lluviosa del 2012 superaron también las del período poco lluvioso de ese año $\left(223.88 \pm 221.61\right.$ cél. $\left.\mathrm{mL}^{-1}\right)$, aunque no se encontraron diferencias estadísticas significativas.

En general, la contribución de los grupos que componen la comunidad fitoplanctónica en ambas épocas climáticas del 2011 y el 2012 mostró un predominio de los flagelados (más del $50 \%)(\mathrm{H}=0.6923)$, con un escaso aporte de las diatomeas $(\mathrm{H}=0.6879)$ y $\operatorname{los}$ dinoflagelados $(\mathrm{H}=0.6857)$ al 


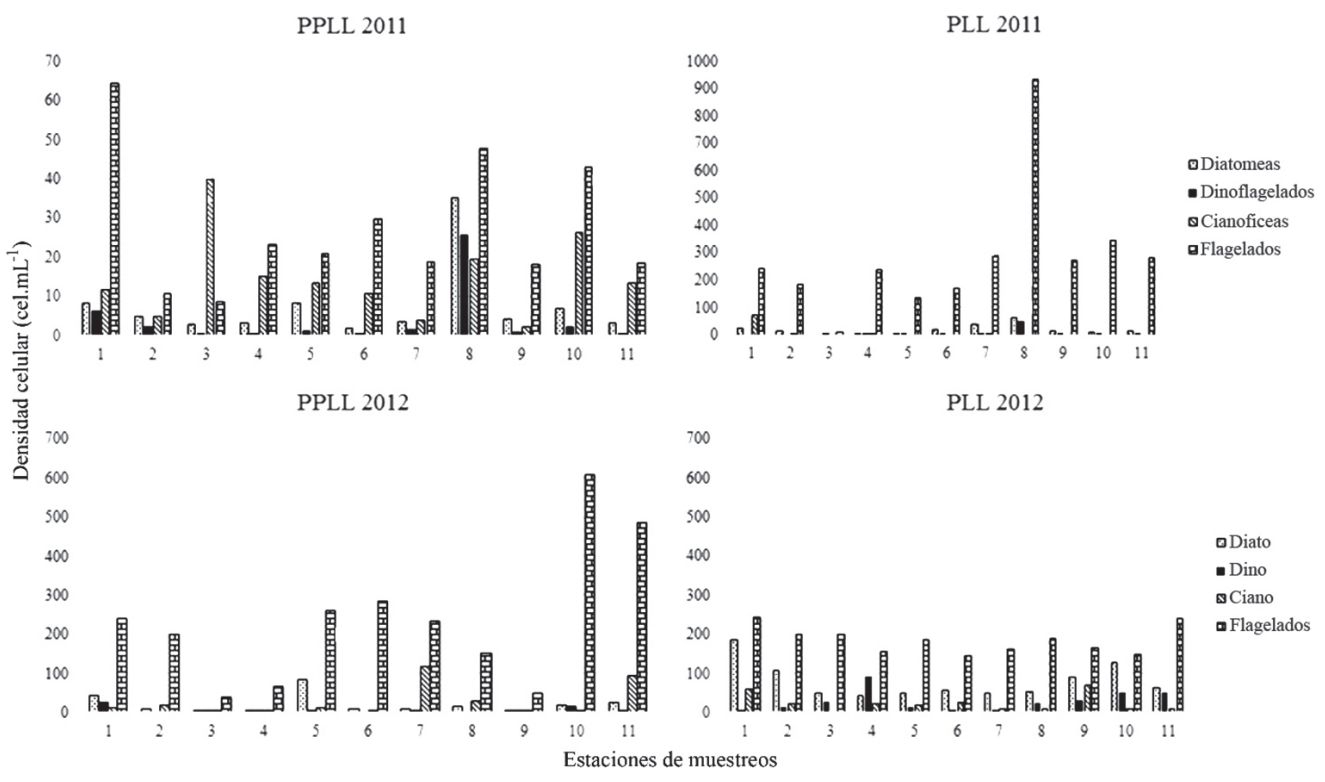

Fig. 3. Contribución de los grupos del fitoplancton a la concentración total por estaciones (PPLL: Período poco lluvioso y PLL: Período lluvioso)

Fig. 3. Contribution of phytoplankton groups to total concentration by station (PPLL: Period of low rainfall and PLL: Rainy period)

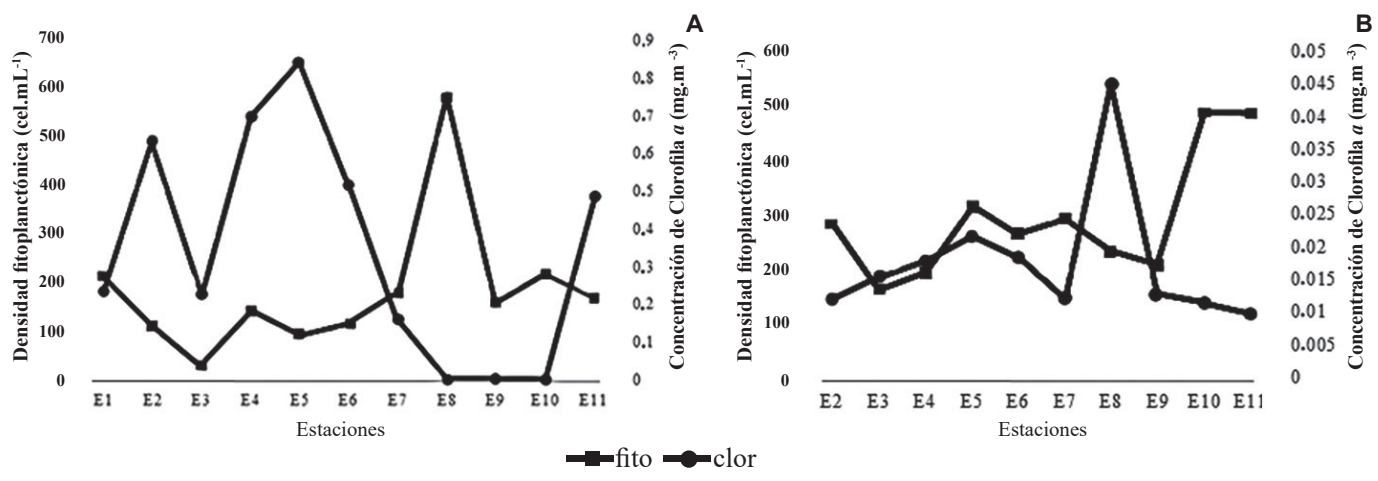

Fig. 4. Comportamiento de la clorofila $a$ y la densidad fitoplanctónica durante los años 2011 (A) y 2012 (B) en ambas temporadas climáticas

Fig. 4. Behavior of chlorophyll $a$ and phytoplankton density during 2011 (A) and 2012 (B) in both climatic seasons

total de la comunidad $(\mathrm{H}=0.6912)$ un incremento en abundancia en el (con menos del 25\%). Aunque en la época lluviosa del 2012 se observó aporte de las diatomeas, este solo llega a representar más del $25 \%$, en solo 
cuatro estaciones: Rincón de Guanabo (E.1), Ranchón de Brisas del Mar (E.2), El Mégano (E.9) y Playa Tarará (E.10) (Fig. 3).

Las cianobacterias presentaron una mayor representatividad en el período poco lluvioso de ambos años; en el caso del año 2012 su mayor representación se observó en la estación correspondiente al Río Itabo (E.7: Mi Cayito) (9\% de la concentración fitoplanctónica total) $(\mathrm{H}$ $=1.186$ ), mientras que en el año 2011 la estación de mayor abundancia del grupo fue la de Guardafronteras (E.3) $(\mathrm{H}=0.7979)$ (Fig. 3, Cuadro 1).

No existió una dominancia (D) en cuanto a la concentración fitoplanctónica entre los grupos (diatomeas, dinoflagelados, cianobacterias, flagelados) que constituyeron la comunidad fitoplanctónica para los meses muestreados, tanto en el año 2011 como en el 2012, al igual que se presentó uniformidad $(\mathrm{J})$ entre las abundancias de cada grupo (Cuadro 2).

Las concentraciones de clorofila $a$ entre ambas épocas resultaron inferiores en el 2012 en relación con el año precedente $\left(\right.$ Xmedia $\left.=0.01 \pm 0.01 \mathrm{mg} \cdot \mathrm{m}^{-3}\right)$. Por otra parte, para ambas épocas en los dos años, no se observó una correspondencia entre los valores de clorofila $a$ y las concentraciones de fitoplancton total por estaciones (Fig. 4 A y B).

\section{Influencia de las variables abióticas}

Las condiciones ambientales abióticas que mayormente están afectando el desarrollo del fitoplancton son la disponibilidad de nitrógeno y fósforo referidas a la relación $\mathrm{NTi} / \mathrm{PO}_{4}$ de acuerdo con el análisis de componentes principales (PCA) (Fig. 5 A y B).

Para el año 2011 no existieron diferencias entre las estaciones, a excepción de Tropicoco (E.8), mientras que las estaciones que difieren del resto de las evaluadas para el año 2012 son Tarará (E.10) y Bacuranao (E.11), siendo estas las que presentaron la mayor densidad fitoplanctónica en ambos años, respectivamente (Fig. 6 A y B), manifestó el Escalado Multidimensional no Métrico (nmMDS). Sin embargo, teniendo en cuenta las variables biológicas (densidad fitoplanctónica) y las variables fisicoquímicas, el ANOSIM reflejó poca diferenciación entre las épocas climáticas tanto para el $2011(\mathrm{R}=0.465)$ como para el $2012(R=0.212)$ y entre estaciones por cada período climático entre ambos años $(R=0.324)$.

Las concentraciones de la DQO, en general, para ambos años evaluados resultaron $<2.0 \mathrm{mg} / \mathrm{L}$ (Cuadro 2); se debe mencionar que solo las estaciones E.5: Calle 476-478, E.8: Hotel Tropicoco y E.10: Playa Tarará presentaron valores de la DQO cercanos al límite inferior de la norma $(>2.0 \mathrm{mg} / \mathrm{L})$.

\section{Evaluación del estado trófico}

La evaluación del estado trófico, según el índice propuesto por Loza (2004), clasifica las aguas de las Playas del Este en el año 2011 como oligotróficas en la época poco lluviosa (febrero); a su vez, alcanzan un nivel 

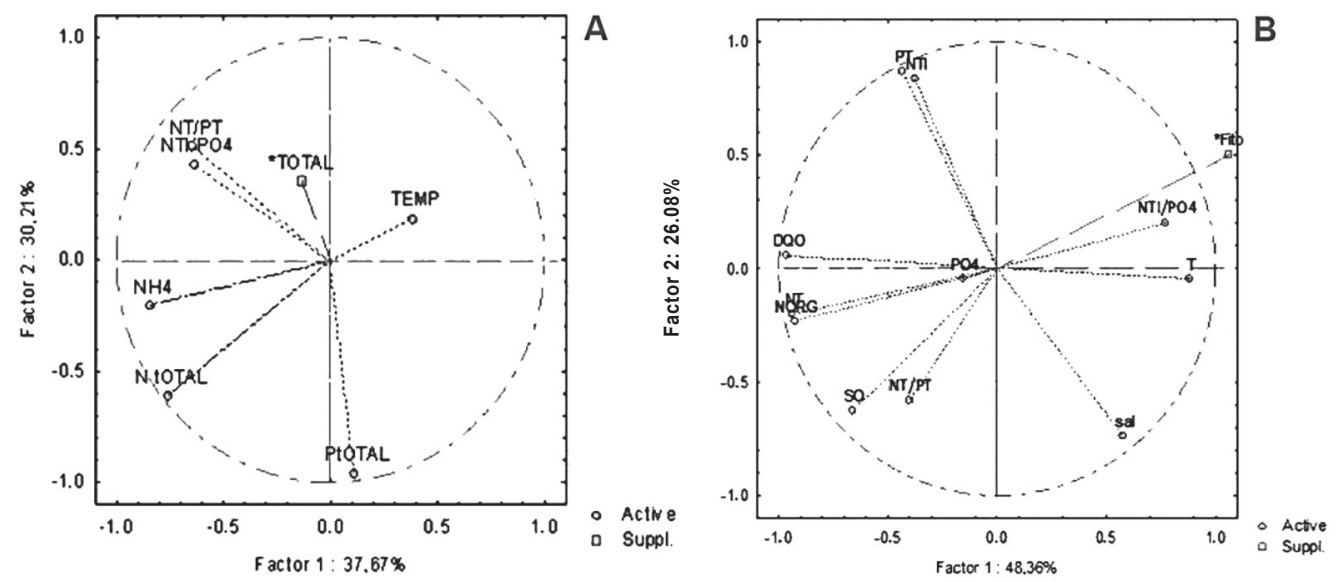

Fig. 5. Análisis de componentes principales para los parámetros químicos en relación con la comunidad de fitoplancton en el 2011 (A) y el 2012 (B) (variable suplementaria: concentración de fitoplancton total)

Fig. 5. Analysis of main components to chemical parameters in connection with the phytoplankton community in 2011 (A) and 2012 (B) (additional variable: concentration of total phytoplankton)
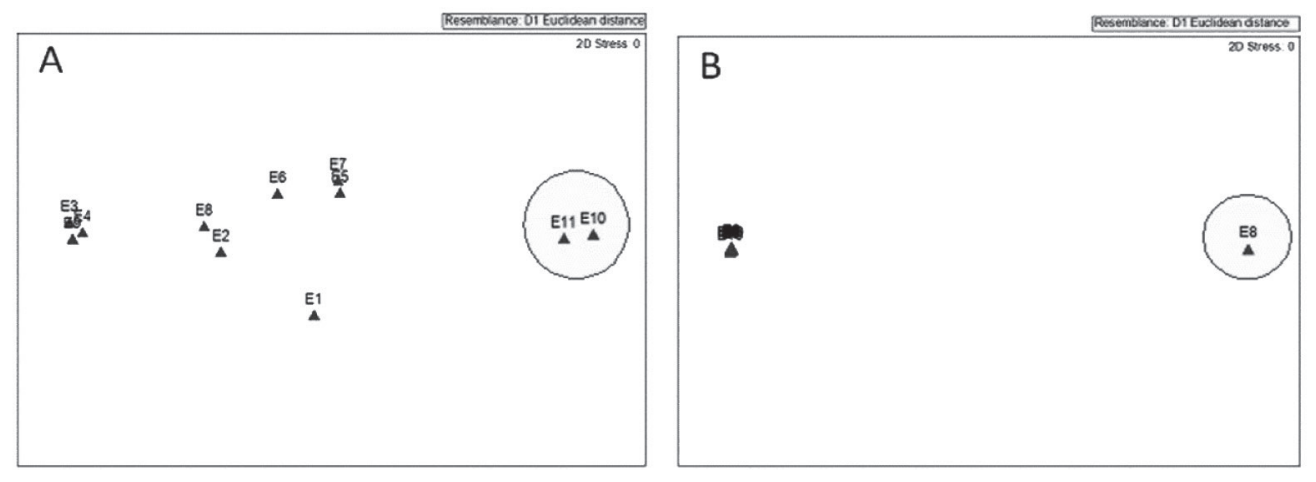

Fig. 6. Escalado Multidimensional no Métrico (nmMDS) entre estaciones de muestreo en ambas temporadas climáticas de los años 2011 (B) y 2012 (A)

Fig. 6. Non-metric multidimensional scaling (nmMDS) between sampling stations in both climatic seasons for 2011 (B) and 2012 (A)

trófico superior (oligomesotróficas) por presentar un estado trófico en la lluviosa (julio). No obstante, superior (Fig. 7A).

existen estaciones como Rincón de En el año 2012, tanto en la Guanabo (E.1), Ranchón de Brisas época poco lluviosa (febrero) como del Mar (E.2), Agro Guanabo (E.4) en la lluviosa (julio), las aguas se y Tropicoco (E.8) que se distinguen clasificaron de forma general como 


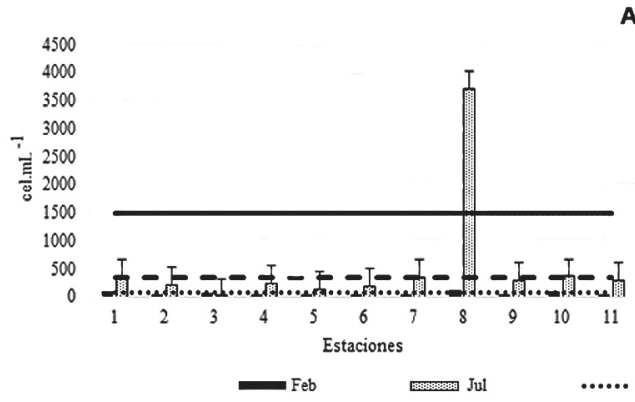

A

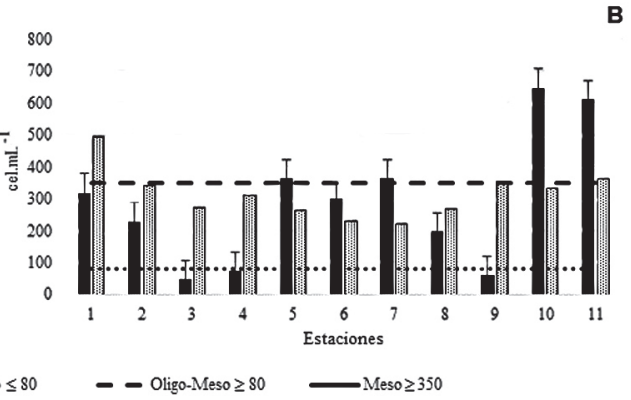

Fig. 7. Estado trófico de las aguas durante los muestreos de 2011 (A) y 2012 (B) (oligotrófico, oligomesotrófico, mesotrófico)

Fig. 7. Trophic status of waters during sampling in 2011 (A) and 2012 (B) (oligotrophic, oligo-mesotrophic, mesotrophic)

oligomesotróficas, con destaque para algunas estaciones con un estado trófico superior (mesotróficas) como Rincón de Guanabo (E.1), Calle 476478 (E.5), Mi Cayito (E.7), Tarará (E.10) y Bacuranao (E.11) (Fig. 7B).

\section{DISCUSIÓN}

El análisis cualitativo de la comunidad fitoplanctónica mostró que el número de taxones de ambos años se mantuvo por debajo a lo reportado por Lugioyo et al. (2009) para esta zona de estudio con valores de 179 taxones, distribuidos en 104 formas de diatomeas, 35 de dinoflagelados, 37 cianobacterias y 3 flagelados. El comportamiento de los taxones en relación con el aporte de los grupos de cianobacterias, flagelados y diatomeas a la comunidad de fitoplancton en el litoral de las Playas del Este, fue similar a lo obtenido por Loza et al. (2007) para las aguas costeras del Golfo de Batabanó, en la región suroccidental de Cuba (flagelados $50 \%$, diatomeas $20 \%$, cianobacterias $30 \%$ ).
Según plantean Loza et al. (2006) y Dobal et al. (2011), en sentido general, en los últimos años se ha detectado un cambio en la estructura de la comunidad de microalgas en las aguas de la plataforma SW, NW y central, con un incremento notable de cianobacterias, organismos indicadores de alteraciones ambientales asociadas a eventos de eutrofización en las zonas costeras.

En el presente estudio se obtuvo una alta representación de cianobacterias en todas las estaciones muestreadas, fundamentalmente en la época de poca lluvia, con un incremento de 13 especies hacia el año 2012 del total de especies encontradas en el 2011, lo que correspondió con elevadas concentraciones de nutrientes y materia orgánica (Cuadro 1).

En las aguas de las Playas del Este estuvieron presentes organismos potencialmente nocivos relacionados con la producción de florecimientos algales en diferentes ecosistemas (Sar et al. 2002), fundamentalmente en las estaciones aledañas a Boca Ciega (E.6), Tarará (E.10) y Bacuranao (E.11). Esto pudo haber estado asociado a una baja 
disponibilidad de nitrógeno inorgánico, pudiendo desarrollarse especies oportunistas como cianobacterias y dinoflagelados. Según Guildford \& Hecky (2000) y Anderson et al. (2002), el desarrollo de especies oportunistas como cianobacterias y dinoflagelados se favorece cuando existe una baja disponibilidad de nitrógeno inorgánico, que para las épocas de poca lluvia en ambos años se obtuvieron menores valores que en la época lluviosa.

El hecho de que se encontraran estos géneros en las Playas del Este constituye una alerta para la vigilancia de la zona, debido al uso de actividades recreativas y pesqueras que tienen estas aguas por la población aledaña. De acuerdo con Sar et al. (2002) y Delgado et al. (2006), estos organismos son susceptibles de aumentar su concentración y provocar cambios de coloración en las aguas; además, tienen la capacidad de producir sustancias tóxicas que pueden afectar la salud de los bañistas por el contacto con la piel y provocar daños en la visión por la acción de los aerosoles, así como intoxicaciones alimentarias.

En general, las concentraciones de fitoplancton encontradas durante el estudio de los años 2011 y 2012 resultaron inferiores a las obtenidas entre los años 2007 y 2009 (Xmáx = 1 656) por Lugioyo et al. (2009) para este sector turístico, en las mismas épocas, lo que responde a los elevados acumulados de lluvias reportados para estos años (112 $\mathrm{mm}$ en el año 2007 y $142 \mathrm{~mm}$ en el año 2009) por el INRH (2007; 2009). El 2012 fue un año más lluvioso (43\% y $85 \%$ ), en ambas épocas, con respecto al 2011, donde solamente en la época lluviosa, el porcentaje de precipitaciones fue elevado (89\%) (INRH, 2011; 2012).

La respuesta de la concentración fitoplanctónica en ambos años reflejó una estrecha relación con el régimen de lluvia, ya que las escorrentías asociadas a este evento meteorológico favorecieron el ingreso de nutrientes desde el río Guanabo hacia la estación de Rincón de Guanabo (E.1), donde se obtuvieron los mayores valores de abundancia de microorganismos en la época lluviosa en el año 2012, mientras que los mayores valores hallados en el año 2011 en la época poco lluviosa en la estación de Tropicoco (E.8) estuvieron ligados a que esta estación se encuentra ubicada hacia el noroeste de la Ensenada de Sibarimar y debido a la acción del viento en las Playas del Este y la presencia de giros de carácter permanente en determinadas áreas del litoral, la dirección de la corriente es de $\mathrm{E}$ a W y paralela a la costa; esto favorece que se concentren los nutrientes y la materia orgánica e inorgánica provenientes de las zonas aledañas hacia las estaciones que se encuentran más al noroeste de la zona costera y, por tanto, se incrementen los contaminantes.

En cuanto a la composición por grupos, predominaron los flagelados y las cianobacterias, los cuales no variaron notablemente en proporción (en el año 2011, 70\% de flagelados y $8 \%$ de cianobacterias; en el año 2012, 84\% de flagelados y $6 \%$ de cianobacterias); por su parte, el aporte de las cianobacterias en el 2012 no 
resultó tan marcado en abundancia como en la época de poca lluvia del año 2011, donde se observó una correspondencia de la respuesta de este grupo ante los valores de nutrientes relacionados con el desbalance en la disponibilidad de $\mathrm{N}$ y $\mathrm{P}$, ya que el aporte de cianobacterias en la época poco lluviosa estuvo asociado a una mayor limitación de $\mathrm{N}$ y de $\mathrm{P}$, con respecto a la época lluviosa.

\section{Las cianobacterias}

son consideradas un adecuado indicador del grado de degradación de las aguas relacionado con un desbalance en la disponibilidad de nitrógeno y fósforo (Bonilla \& Conde, 1998). El desarrollo de las cianobacterias en la época poco lluviosa del 2011 pudo estar favorecido por la existencia de un desequilibrio de estos compuestos. Lo anterior se pudo corroborar por los valores de las relaciones $\mathrm{NTi} / \mathrm{PO}_{4}$ y NT/PT (Cuadro 2) encontrados en las dos épocas del 2011, que mostraron la existencia en febrero de condiciones limitantes de $\mathrm{N}$ en el $73 \%$ de las estaciones y de $\mathrm{P}$ en el $45 \%$ de las estaciones, con una carencia de $\mathrm{N}$ tres veces mayor y de $\mathrm{P}$ dos veces mayor, en relación con la época lluviosa del año 2011.

Lo anterior sustenta lo informado por Contreras-Espinosa et al. (1996) y Guildford \& Hecky (2000) con respecto a las condiciones de limitación de $\mathrm{N}$ y $\mathrm{P}$ en el medio que favorecen el desarrollo de organismos oportunistas como las cianobacterias. Estos autores establecen que cuando los valores de la relación NT/PT son superiores a 50 indican una tendencia a la carencia de fósforo y que cuando los estimados de la razón $\mathrm{NTi} / \mathrm{PO}_{4}$ son inferiores a 15 , el nitrógeno se considera la fuente de nutriente limitante en el metabolismo del fitoplancton.

Los valores de la DQO, en general, para ambos años evaluados reflejan una adecuada calidad de las aguas en cuanto a la contaminación por materia orgánica; las estaciones E.5: Calle 476-478, E.8: Hotel Tropicoco y E.10: Playa Tarará presentaron valores de la DQO cercanos al límite inferior de la norma $(>2.0 \mathrm{mg} / \mathrm{L})$ establecida por la EPA (Inoue \& Ebise, 1991) para ser considerada un agua marina contaminada por materia orgánica.

Otro indicador fitoplanctónico usualmente empleado paramedir de forma indirecta la biomasa fitoplanctónica y el estado trófico de los sistemas acuáticos es la concentración de clorofila $a$ (ContrerasEspinosa et al. 1994). En el caso de las Playas del Este no se encontró una correspondencia entre la clorofila $a$ y las mayores concentraciones de fitoplancton; esto se pudo deber a que la concentración de pigmentos fotosintéticos puede variar, tanto entre especies como dentro de una misma célula, en dependencia de la salinidad, intensidad de luz incidente, temperatura y estado fisiológico de las células y el tamaño de los organismos (Regadera, 2006).

El análisis de componentes principales (PCA) con los datos de las diferentes variables fisicoquímicas, la clorofila a y como variable suplementaria la concentración de fitoplancton, reflejaron que existe una alta influencia entre las 
concentraciones de fitoplancton y la disponibilidad de nitrógeno y fósforo referidas a la relación $\mathrm{NTi} / \mathrm{PO}_{4}$. Lo anterior corrobora lo informado por Corredor et al. (1999) sobre la gran influencia que tienen el nitrógeno y el fósforo en la productividad biológica y en la estructura de los ecosistemas marinos tropicales.

El Escalado Multidimensional no Métrico (nmMDS), tomando en cuenta las diferentes variables fisicoquímicas y biológicas, mostró que las estaciones Tarará (E.10) y Bacuranao (E.11) difieren del resto de las evaluadas, con una mayor similitud de las estaciones en el año 2011, donde solo la estación correspondiente al Hotel Tropicoco (E.8) se diferencia de las demás. Ello podría asociarse a la ubicación geográfica de estas estaciones, ubicadas al extremo oeste de la Ensenada de Sibarimar, constituyendo zonas de máxima deriva hacia la costa.

En las Playas del Este, es de esperarse una deriva de $\mathrm{E}$ a $\mathrm{W}$ con fluctuaciones locales significativas en direcciones $\mathrm{N}, \mathrm{NW}, \mathrm{S}$ y $\mathrm{SE}$, específicamente en las inmediaciones de las estaciones Tropicoco (E.8), Agro Guanabo (E.4) y Guardafronteras (E.3) (Lugioyo et al. 2013). Ello propicia el aumento de concentraciones de nutrientes y organismos planctónicos hacia esa zona.

La respuesta del fitoplancton determina el estado trófico del ecosistema $\mathrm{y}$ en correspondencia se obtuvo un incremento en el nivel trófico asociado al régimen de lluvias y a la temperatura del agua. Lo anterior se pudo confirmar por las características tróficas encontradas en la época lluviosa y la poco lluviosa de ambos años, donde, en general, a partir del fitoplancton, las aguas presentaron características oligomesotróficas con algunas estaciones mesotróficas en el año 2012, con respecto al año 2011, lo que resultó similar a las características tróficas encontradas durante el año 2007 y el año 2009 por Lugioyo et al. (2009).

Según Ruiz \& Delgado (2012), la calidad ambiental de la zona costera de las Playas del Este se ha visto afectada por residuos líquidos que llegan a la costa procedentes de las escorrentías superficiales del área litoral, los arrastres de las cuencas fluviales y el manejo inadecuado de las aguas residuales urbanas que provienen de los sectores residencial y turístico, así como por el impacto del gran número de bañistas que arriban a las playas, sobre todo en la época de verano, con una afluencia de más de 120000 bañistas/día los fines de semana. En este sentido, la calidad del agua se encuentra contaminada por vertido de residuales de instalaciones turísticas, zonas de viviendas, actividades productivas industriales y agropecuarias; existen problemas higiénico-sanitarios en zonas puntuales motivados por la presencia de fosas, tanques sépticos comunes y pozos de infiltración en mal estado, además de los residuales que corren libremente por las calles a través de zanjas que vierten directamente a la zona de playa.

El aumento en el nivel trófico de estas estaciones está relacionado con el 
enriquecimiento de nutrientes, asociado al régimen de precipitaciones de ambos años, como se observa por las elevadas concentraciones de nitrógeno total inorgánico. Se debe prestar atención a las condiciones ambientales existentes en la estación de Rincón de Guanabo, si consideramos que ha podido ocurrir en los últimos tiempos un incremento de las descargas de desechos domésticos por el establecimiento y uso de cafeterías por parte de los pobladores.

\section{CONCLUSIONES}

Las aguas de las Playas del Este durante los años 2011 y 2012 presentaron una calidad moderada según la concentración de la comunidad fitoplanctónica, con un estado intermedio entre condiciones oligotróficas y oligomesotróficas.

La presencia de cianobacterias $y$ de organismos nocivos en ambos años, en el período poco lluvioso, es un indicativo de la ocurrencia de estrés ambiental en esta zona costera, asociado a concentraciones de nutrientes y materia orgánica que pueden estar afectando la zona de manera puntual.

Las concentraciones de clorofila $a$ en los dos meses muestreados de ambos años fueron inferiores a las encontradas en años anteriores y los mayores valores se hallaron en el período lluvioso del año 2012, siendo Tarará (E.10) la estación de mayor concentración del pigmento.

Las condiciones ambientales abióticas que están afectando el desarrollo del fitoplancton en los meses muestreados en las Playas del Este son la disponibilidad de nitrógeno y fósforo referidas a la relación $\mathrm{NTi} / \mathrm{PO}_{4}$.

El aumento en el nivel de concentración fitoplanctónica de estas estaciones está relacionado con el enriquecimiento de materia orgánica, asociado al régimen de precipitaciones de ambos años.

\section{AGRADECIMIENTOS}

Agradecemos a los revisores por todos los señalamientos realizados al artículo, ya que ayudaron a mejorar la calidad del trabajo.

\section{BIBLIOGRAFÍA}

Achá, D. \& Fontúrbel, F. (2003). La diversidad de una Comunidad: ¿Está controlada por Top - Down, Bottom - Up o una combinación de estos? Rev. Biol. Org., 13, 1-16.

Anderson, D. M., Glibert, P. M. \& Burkholder, J. M. (2002). Harmful algal blooms and eutrophication: $\mathrm{Nu}-$ triens sources, composition, and consequences. Estuares, 25(4b), 707-726.

Barron, J. A. \& Baldauf, J. G. (1989). Tertiary cooling steps and paleproductivity as reflected by diatoms and biosiliceous sediments. In W. H. Berger, V. S. Smetacek \& G. Wefer (Eds.), Productivity of the oceans: present and past (pp. 341-354). New York, EE. UU.: Wiley-Interscience.

Bonilla, S. \& Conde, D. (1998, noviembre). El fitoplancton como descriptor sensible de cambios ambientales en las lagunas costeras de la reserva $\mathrm{Ba}$ ñador del Este. Ponencia presentada en Seminario-Taller sobre monitoreo ambiental. Rocha, Uruguay. 
Cabrera, R. \& Suárez, A. M. (2003). Adiciones a las clorofíceas de Cuba del género Avrainvillea Decaisne, 1842 (Bryopsidales, Udoteaceae). Rev. Invest. Mar., 24(2), 95-98.

Contreras-Espinosa, F., Castañeda-López, O. \& García-Nagaya, A. (1994). La clorofila $a$ como base para un índice trófico en lagunas costeras mexicanas. An. Inst. Cienc. Mar. Limnol., 21, 55-66.

Contreras, F., Castañeda, O., Torres-Alvarado, R. \& Gutiérrez, M. F. (1996). Nutrientes en 39 lagunas costeras mexicanas. Rev. Biol. Trop., 44(2), 417-425.

Corredor, J. E., Howarth, R. W., Twilley, R. R. \& Morell, J. M. (1999). Nitrogen cycling and anthropogenic impact in the tropical Inter-American seas. Biogeochemistry, 46, 163-178. http:// dx.doi.org/10.1007/BF01007578

De la Lanza-Espino, G., Hernández-Pulido, S. \& Carvajal-Pérez, J. L. (2000). Organismos indicadores de la calidad del agua y de la contaminación (bioindicadores). México: Plaza y Valdés.

Delgado, G., Lechuga-Devéze, C. H., Popowski, G., Troccoli, L. \& Salinas, C. A. (2006). Epiphytic dinoflagellates associated with ciguatera in the northwestern coast of Cuba. Rev. Biol. Trop., 54(2), 29-310.

Dobal, V., Loza, S. \& Lugioyo, M. (2011). Potencialidades de las cianobacterias planctónicas como bioindicadores de estrés ambiental en ecosistemas costeros. Serie Oceanológica, 9, 112-117. Ebenezer, V., Medlin, L. K. \& Ki, J. (2012). Molecular Detection, Quantification, and Diversity Evaluation of Microalgae. Mar. Biotechnol., 14(2), 129-142. http://dx.doi.org/10.1007/ s10126-011-9427-y

Fretwell, S. D. \& Barach, A. L. (1977). The regulation of plant communities by food chains exploiting them. Perspectives in biology and medicine, 20(2), 169-185. http://dx.doi.org/10.1353/pbm.1977.0087

Frontier, S. (1981). Recuentos y análisis de los datos. Tratamiento de los datos. En D. Boltovskoy (Ed.), Atlas del zooplancton del Atlántico Sudoccidental $y$ método de trabajo con zooplancton marino (pp. 169-188). Mar del Plata, Argentina: INIDEP.

Gamboa-Márquez, J. F., Sánchez-Suárez, I. G. \& La Barbera-Sánchez, A. (1994). Dinoflagelados (Pyrrophyta) del Archipiélago Los Roques (Venezuela): Familias Prorocentraceae y Ostreopsidaceae. Acta Cient. Venezolana, 45(2), 140-152.

Gregor, J. \& Marsálek, B. (2004). Freshwater phytoplankton quantification by chlorophyll $a$ : a comparative study of in vitro, in vivo and in situ Methods. Water Res., 38(3), 517-522.

Guildford, S. J. \& Hecky, R. E. (2000). Total nitrogen, total phosphorus, and nutrient limitation in lakes and oceans: Is there a common relationship? Limnol. Oceanogr., 45(6), 1213-1223. http:// dx.doi.org/10.4319/lo.2000.45.6.1213

Guiry, M. D. \& Guiry, G. M. (2012). AlgaeBase. World-wide electronic publication, National University of Ireland, Galway. Recuperado en enero 15, 2011, disponible en http://www.algaebase.org/

Hammer, O., Harper, D. A. T. \& Ryan P. D. (2001). PAST: Paleontological statistics software package for education and data analysis, version 3.1. Oslo, Noruega. http://palaeo-electronica. org/2001_1/past/issue1_01.htm

Hasle, G. R. \& Syvertsen, E. E. (1996). Marine diatoms. In C. R. Tomas (Ed.), Identifying Marine Diatoms and Dinoflagellates (pp. 5-385). San Diego, EE. UU.: Academic Press. 
Inoue, T. \& Ebise, S. (1991). Runoff characteristics of COD, BOD, C. N. and P loading from rivers to enclosed coastal seas. Mar. Pollut. Bull., 23, 11-14.

INRH. Instituto Nacional de Recursos Hidráulicos. (2007). Análisis de lluvia, cuencas hidrográficas en las Playas del Este. Boletín Hidrológico. Revisión en 15 diciembre, 2007, disponible en http://www.hidro.cu/cuba.html

INRH. Instituto Nacional de Recursos Hidráulicos. (2009). Análisis de lluvia, cuencas hidrográficas en las Playas del Este. Boletín Hidrológico. Revisión en 12 diciembre, 2009, disponible en http://www.hidro.cu/cuba.html

INRH. Instituto Nacional de Recursos Hidráulicos. (2011). Análisis de lluvia, cuencas hidrográficas en las Playas del Este. Boletín Hidrológico. Revisión en 8 diciembre, 2011, disponible en http:// www.hidro.cu/cuba.html

INRH. Instituto Nacional de Recursos Hidráulicos. (2012). Análisis de lluvia, cuencas hidrográficas en las Playas del Este. Boletín Hidrológico. Revisión en 10 diciembre, 2012, disponible en http://www.hidro.cu/cuba.html

Koblentz-Mishke, O. J. \& Vedernikov, V. I. (1979). Producción Primaria. En M. E. Vinogradov (Ed.), Oceanología, Biología del Océano (pp. 183-309). Nauka, Moscú: Producciones Biológicas del Océano.

Licea, S., Moreno, J. L., Sant, H. \& Figueroa, G. (1995). Dinoflagelados del Golfo de California. México: Univ. Auton. de Baja California Sur.

Loza, S., Barrios, K., Miravet, M. E., Lugioyo, M., Perigó, E. \& Sánchez, M. (2003). Respuesta del fitoplancton ante el estrés ambiental en los ecosistemas arrecifales. Protección del Hombre y la Naturaleza. Cátedra de Educación Ambiental, 8, 21-24.
Loza, S. (2004). Índices de calidad y estado trófico de las aguas. En M. E. Miravet, M. Lugioyo, S. Loza, D. Enríquez, Y. Delgado, M. Carmenate \& D. M. Pérez (Eds.), Procedimientos para el monitoreo de la calidad ambiental en la zona marino costera a partir de microorganismos (pp. 76-84). La Habana, Cuba: Centenario.

Loza, S., Montalvo, J. F., Cantelar, K., Del Valle, R., Sánchez, M., García, I. ... \& Esquivel, M. (2006). Diagnóstico de la situación ambiental existente en la bahía de Jigüey (NE de Cuba) en relación con la problemática de intoxicación alimentaria de origen marino. Archivo Científico Inst. Oceanología. La Habana, Cuba: Centenario.

Loza, S., Lugioyo, M., Martínez, M., Miravet, M. E., Montalvo, J. \& Sánchez, M. (2007). Evaluación de la calidad de las aguas del Golfo de Batabanó a partir de indicadores biológicos y químicos. Rev. Invest. Mar., 28(2), 111-120.

Lugioyo, M., Loza, S., Arriaza, L., Enríquez, D., Castellanos, S., Delgado, Y., Carmenate, M., Sánchez, M., Pérez, D. M., García, R., Núñez, R. \& Reyes, T. (2009). Evaluación de la calidad biológica de las aguas y los sedimentos de una zona costera de importancia turística: Playas del Este. Archivo Científico Inst. Oceanología. La Habana, Cuba: Centenario.

Lugioyo, M., Loza, S., Arriaza, L., Enríquez, D., Castellanos, S., Delgado, Y., Carmenate, M., Sánchez, M., Pérez, D. M., García, R., Núñez, R. \& Reyes, T. (2013). Validación de indicadores microbiológicos para la evaluación de la calidad de las aguas y los sedimentos de una zona costera de importancia turística: Playas del Este. Archivo Científico Inst. Oceanología. La Habana, Cuba: Centenario. 
MapInfo, Corp. (2001). MapInfo Profesional: User's Guide, MapInfo Corporation, version 5.5 www.mapinfo.com. New York, EE. UU.: MapInfo Corp.

Miravet, M. E., Lugioyo, M., Loza, S., Enríquez, D., Delgado, Y., Carmenate, M. \& Pérez, D. M. (2009). Procedimientos para el monitoreo de la calidad ambiental en la zona marino costera a partir de microorganismos. La Habana, Cuba: Centenario.

Montalvo, J. F., García, I de los A., Perigó, E., Alburquerque, O. C., López, D., Betanzos, A. J., Almeida, M. \& García, N. (2013). Calidad química del agua y sedimento en las bahías del Archipiélago Sabana - Camagüey. Rev. Cubana de Quím., 25(2), 123-133.

Moreira, A., Seisdedo, M., Leal, S., Comas, A., Delgado, G., Regadera, R., Alonso, C., Muñoz, A. \& Abatte, M. (2007). Composición y abundancia del fitoplancton de la bahía de Cienfuegos, Cuba. Investig. Mar., 28(2), 97-109.

Moreno, J., Licea, S. \& Santoyo, H. (1996). Diatomeas del Golfo de California. Secretaría de Educación Pública, Fondo para Modernización de la Educación Superior, Programa Interdisciplinario e Interinstitucional Mar de Cortés. México: Univ. Auton. de Baja California Sur.

Pinto, A. M. F., von Sperling, E. \& Moreira, R. M. (2001). Chlorophyll- $a$ Determination Via Continuous Measurement of Plankton Fluorescence: Methodology Development. Water Res., 35(16), 3977-3981. http://dx.doi. org/10.1016/S0043-1354(01)00102-6

Popowski, G. C. \& Borrero, N. A. (1989). Utilización de fijadores en la conservación de flagelados y su influencia en la determinación de la concentración de fitoplancton en el Golfo de Batabanó, Cuba. Rev. Invest. Oceanol., 11, 1-8.

Regadera, R. (2006). Fitoplancton y clorofila a como indicadores de eutrofización en la bahía de La Habana. Tesis de Maestría no publicada, Universidad de La Habana, Cuba.

Ruiz, J. D. \& Delgado, J. J. (2012). La degradación ambiental de los paisajes de las cuencas Guanabo - Itabo. Perspectivas y metodologías de análisis para el desarrollo territorial sostenible de Playas del Este, Cuba. La Habana, Cuba: Académica Española. http://www.alparimedia.com/2016/05/25/la-degradacinambiental-de-los-paisajes-de-las-cuencas-guanabo-itabo-kn2/ o http://www. energydrinkband.com/2016/05/29/la-degradacin-ambiental-de-los-paisajes-delas-cuencas-guanabo-itabo-668/

Sant'Anna, C., Azevedo, M. T., Agujaro, L., Carvalho, M. C. \& Souza, R. C. (2005). Manual Ilustrado Identificação e Contagem de Cianobactérias Planctônicas de Águas Continentais Bras. Sao Paulo, Brasil: Interciencia Press.

Sar, E., Ferrario, M. E. \& Beatriz, R. (2002). Floraciones algales nocivas en el Cono Sur Americano. Madrid, España: Instituto Español de Oceanografía. Sosa, M., Rivas, L., Guerra, R., Felipe, M. \& García, R. (2005). Análisis actual de los procesos erosivos en las Playas del Este de Ciudad de La Habana. Informe inédito. Archivo Científico Inst. Oceanología. La Habana, Cuba: Centenario.

STATSOFT, Inc. (2001). Statistics for Windows (Computer Program Manual), version 6. www.statsoft.com. Tulsa, EE. UU.: Statsoft, Inc.

Tomas, C. R. (1997). Identifying Marine Diatoms and Dinoflagellates. New York, EE. UU.: Academic Press.

Vaulot, D., Eikrem, W., Viprey, M. \& Moreau, H. (2008). The diversity of small eukaryotic phytoplankton $(<3 \mu \mathrm{m})$ in marine ecosystems. FEMS Microbiol. Rev., 32, 795-820. http://dx.doi. org/10.1111/j.1574-6976.2008.00121.x 